\title{
Grazing-incidence x-ray diffraction study of pentacene thin films with the bulk phase structure
}

$\operatorname{AUTHOR}(S)$ :

Yoshida, H; Sato, N

\section{CITATION:}

Yoshida, $\mathrm{H}$... [et al]. Grazing-incidence x-ray diffraction study of pentacene thin films with the bulk phase structure. APPLIED PHYSICS LETTERS 2006, 89(10): 101919.

\section{ISSUE DATE:}

2006-09-04

URL:

http://hdl.handle.net/2433/50335

\section{RIGHT:}

Copyright 2006 American Institute of Physics. This article may be downloaded for personal use only. Any other use requires prior permission of the author and the American Institute of Physics. 


\title{
Grazing-incidence x-ray diffraction study of pentacene thin films with the bulk phase structure
}

\author{
Hiroyuki Yoshida a) and Naoki Sato \\ Institute for Chemical Research, Kyoto University, Uji, Kyoto 611-0011, Japan
}

(Received 22 May 2006; accepted 18 July 2006; published online 8 September 2006)

The structure of pentacene thin films in the bulk phase having interplane spacing of $d(001)$ $=1.44 \mathrm{~nm}$ grown on $\mathrm{SiO}_{2}$ was investigated using grazing-incidence $\mathrm{x}$-ray diffraction. The films were prepared with two different methods: one treated with an organic solvent after vacuum deposition and the other thermally treated. Both films are similar in structure to the single crystal reported by Campbell et al. [Acta Crystallogr. 15, 289 (1962)]. () 2006 American Institute of Physics.

[DOI: $10.1063 / 1.2349307]$

Pentacene thin films are extensively studied as a functional layer of organic field-effect transistors. The structure of the thin films is crucial to understand their transport properties, since charge carriers in them move from one molecule to another along the $\pi$ stacking direction. ${ }^{1}$ However, the structure of pentacene thin films is still not fully understood because of the existence of several polymorphs depending on their preparation conditions, e.g., film thickness, substrate, and temperature. ${ }^{2}$

Thin films of pentacene grown on $\mathrm{SiO}_{2}$ by vacuum deposition technique are particularly notable because most devices are fabricated on $\mathrm{SiO}_{2}$ substrates. At least two polymorphs are reported. ${ }^{2,3}$ Since full analysis of the structures has not been made, they are identified by their interplane spacing along the $(00 l)$ direction, as measured with x-ray diffraction. One is a "thin film" phase with $d(001)$ $=1.54 \mathrm{~nm}$, usually obtained from vacuum deposition to the thickness lower than critical thickness (around $50 \mathrm{~nm}$ ). Another is "bulk phase" with $d(001)=1.44 \mathrm{~nm}$, which is often observed on top of the thin film phase in thicker films. ${ }^{3}$ Both structures are again different from the most frequently cited structure of the single crystal, which has an interplane spacing of $d(001)=1.41 \mathrm{~nm}$.

The thin film phase is known to be irreversibly transformed to the bulk phase by either heat treatment ${ }^{2}$ or immersion in an organic solvent. ${ }^{6}$ Such transitions from a metastable polymorph to a more stable one are frequently observed for organic solids. The bulk phase can therefore be considered as a stable form, while the thin film phase is metastable. $^{2}$

The structure of the bulk phase on $\mathrm{SiO}_{2}$ has not perfectly been identified. Using powder x-ray diffraction, Mattheus et al. analyzed a bulk phase film prepared with deposition on $\mathrm{SiO}_{2}$ followed by treatment of an organic solvent and obtained cell parameters. ${ }^{2,5}$ On the other hand, it seems to be widely believed that the structure of the bulk phase film is the same as a single crystalline structure reported by Campbell et al., ${ }^{7}$ on the basis of the lattice spacing of $d(001)$ $=1.45 \mathrm{~nm}$ almost the same as those of the bulk phase films. However, this has not yet been confirmed experimentally as far as we know. It should be noted that most of polymorphs of pentacene thin films are identified only by interplane

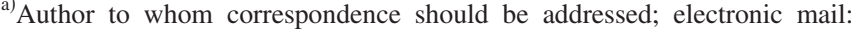
yoshida@e.kuicr.kyoto-u.ac.jp
}

$d(001)$ spacing. Moreover, we should bear in mind that two thin films with the same $d(001)$ do not necessarily have the same structure, i.e., cell parameters and molecular arrangements in the unit cells.

The different interpretations arise from the disuse of suitable experimental methods to determine the structure of a thin film. Among the methods to obtain structural data other than the $d(001)$ interplane spacing, electron diffraction has most frequently been used for pentacene thin films, though the number of usable substrates is limited. ${ }^{8-10}$ Recently, grazing-incidence $\mathrm{x}$-ray diffraction (GIXD) has been applied to mono- or multilayer thin films of pentacene grown on bare or self-assembled monolayer-treated $\mathrm{SiO}_{2}$ substrates. ${ }^{11-13} \mathrm{In}$ this technique an x-ray beam incident on a sample film at a grazing angle less than the critical angle of total reflection enables us to acquire information on lattice spacings parallel to the sample surface. The obtained diffraction pattern includes more information than the specular angle x-ray diffraction one for organic thin films that usually show only a few peaks.

In this letter, we apply GIXD to investigate the structure of the bulk phase pentacene films grown on $\mathrm{SiO}_{2}$ substrate. The films were prepared with two different methods. We present a simple model to simulate the GIXD pattern in accordance with a reciprocal lattice map. The observed diffraction patterns turn out to show good correspondence with the single crystal structure reported by Campbell et al.

Purified pentacene was purchased from Tokyo Kasei Kogyo Co. Ltd. and used without further purification. $\mathrm{Si}(100)$ substrates were washed in acetone and de-ionized water, and finally treated by UV ozone for $2 \mathrm{~h}$. Two different methods were used to obtain pentacene films in the bulk phase. In one method, a $50 \mathrm{~nm}$ thick pentacene thin film was deposited at $0.1 \mathrm{~nm} \mathrm{~s}^{-1}$ on a substrate at room temperature, and the specimen was dipped in acetone for $1 \mathrm{~h}$. In the other method, a $120 \mathrm{~nm}$ thick film was deposited at $0.2 \mathrm{~nm} \mathrm{~s}^{-1}$ on a $40{ }^{\circ} \mathrm{C}$ substrate, and the specimen was heated to $150{ }^{\circ} \mathrm{C}$ in vacuum and slowly cooled down for about $12 \mathrm{~h}$. During the vacuum depositions and the heat treatment the ambient pressures were kept lower than $1 \times 10^{-4} \mathrm{~Pa}$.

All the x-ray diffraction measurements were carried out on a thin film X-ray diffractometer (Rigaku, ATX-G). X-ray $(\mathrm{Cu} K \alpha, \lambda=0.154184 \mathrm{~nm})$ beam was monochromatized and collimated with a multilayer x-ray mirror and incident Soller slits of $0.48^{\circ}$ in the divergence angle. The diffracted beam 


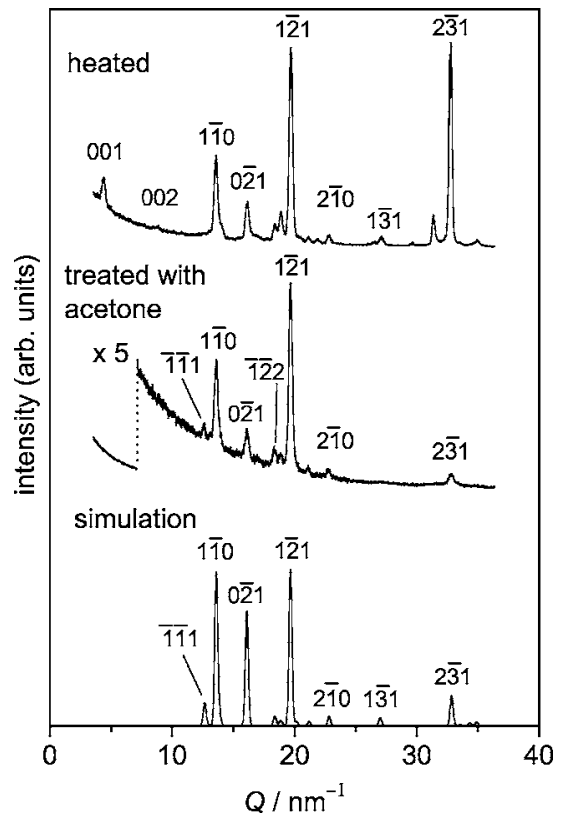

FIG. 1. Grazing-incidence $x$-ray diffraction patterns of pentacene thin films in the bulk phase, prepared with thermal (upper) and acetone (middle) treatments of vacuum deposited sample. Peaks are indexed in accordance with the simulation (lower) based on the crystal structure (Ref. 7).

for detection was also collimated with receiving Soller slits with $0.41^{\circ}$ in the divergence angle.

The specular angle $x$-ray diffraction patterns of the acetone-treated thin film show only a peak at $d(001)$ $=1.44 \mathrm{~nm}$, while the thermally treated thin film demonstrates peaks at both 1.44 and $1.54 \mathrm{~nm}$. It is therefore necessary to extract diffraction signals for the bulk phase from the observed pattern corresponding to the mixture of the two phases in the thermally treated sample.

In-plane GIXD patterns of the bulk phase pentacene films are shown in Fig. 1. The pattern simulated in accordance with the crystal structure reported by Campbell et $\mathrm{al}^{7}$ was used to index the observed peaks, as will be mentioned later. The diffraction pattern of the acetone-treated film was measured at an incident angle of $\omega=0.17^{\circ}$ to achieve maximum intensity.

For the thermally treated film, $\omega=0.13^{\circ}$ was chosen to gain surface diffraction intensity to eliminate a diffraction signal from the thin film phase underneath; in the thermally treated film, layers of the bulk phase appear to grow on top of the thin film phase. ${ }^{3}$ In GIXD, the penetration depth $t$ of incident $\mathrm{x}$-ray beam is approximated to be $\lambda /\left\{2 \pi\left(\theta_{c}^{2}\right.\right.$ $\left.\left.-\omega^{2}\right)^{1 / 2}\right\}$ where $\lambda$ is the $\mathrm{x}$-ray wavelength and $\theta_{c}$ is the critical angle for the total reflection. ${ }^{14,15}$ As $\theta_{c}$ for pentacene was calculated to be $0.17^{\circ}$, diffraction pattern of the top $t \approx 14 \mathrm{~nm}$ can be measured at $\omega=0.13^{\circ}$.

In the GIXD patterns, the bulk phase and the thin film phase show peaks at $Q=16.1$ and $16.6 \mathrm{~nm}^{-1}$, respectively. The diffraction pattern for $\omega=0.17^{\circ}$ shows both peaks at 16.1 and $16.6 \mathrm{~nm}^{-1}$. The intensity for the thin film phase becomes lower at smaller $\omega$, and finally negligible at $\omega=0.13^{\circ}$. Thus the diffraction pattern for only the bulk phase of the thermally treated sample was obtained at $\omega=0.13^{\circ}$.

To examine the orientation of the $c^{*}$ axis, we carried out the $\omega$ scan and the pole figure measurement of (001) diffractions for both samples. As a result, the $c^{*}$ axis was found surface normal. Any differences were below the detection Downloaded 05 Mar 2008 to 130.54.110.22. Redistribution subject

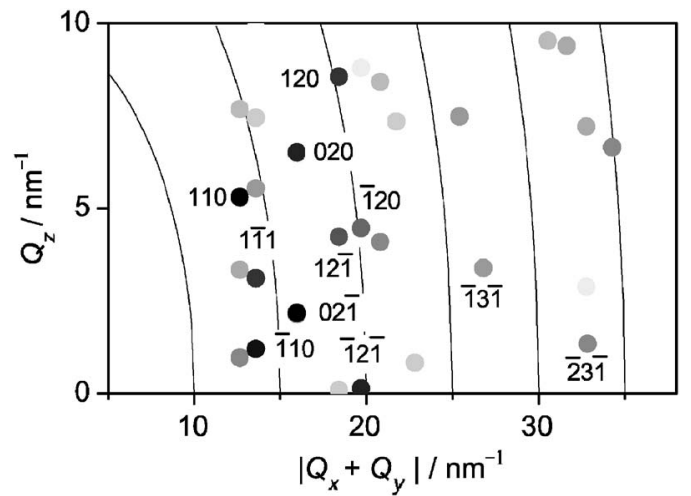

FIG. 2. Reciprocal lattice mapping based on the structure by Campbell et al. (Ref. 7). Diffraction intensity is indicated by the darkness of the point.

limits imposed by our experimental resolution and sensitivity. On the other hand, no preferential orientation was found around the $c^{*}$ axis from the $\phi$ scan for (1-10) diffraction.

As the lattice orientations in the pentacene films are obtained, the in-plane diffraction pattern is simulated using the crystal structure data reported by Campbell et al. ${ }^{7}$ to identify the structure of our bulk phase films. In the in-plane diffraction pattern, the peak positions are determined by cell parameters, and the peak intensities are primarily governed by the molecular arrangement (position of atoms in the unit cell) and orientation of the unit cell. We consider $\mathrm{x}$-ray diffraction from three-dimensional pentacene crystals closely arranged with their $c^{*}$ axes normal and with their random orientation around each $c^{*}$ axis. A set of reciprocal lattice vectors, $\mathbf{G}$ $=h \mathbf{a}^{*}+k \mathbf{b}^{*}+l \mathbf{c}^{*}$, was chosen in accordance with the reported cell parameters. For each reciprocal lattice vector, diffraction intensity, $I_{0}(h, k, l)$, was calculated using a standard software for powder x-ray pattern analysis. ${ }^{16}$ As shown in Fig. 2, the reciprocal lattice points and their diffraction intensities are mapped on two-dimensional surface of $\left|Q_{x}+Q_{y}\right|$ and $Q_{z}$, where $\mathbf{G}=\left(Q_{x} / 2 \pi, Q_{y} / 2 \pi, Q_{z} / 2 \pi\right)$ with the $z$ axis parallel to the $c^{*}$ axis. As the sample surface is in the $x y$ plane, reciprocal lattice points only at $Q_{z}=0$ should be observed in the in-plane pattern of GIXD.

In the actual experiment, however, diffraction peaks for $Q_{z}$ close to zero are also detected, probably due to the instrumental resolution, crystal domain sizes, distribution of crystal orientations, etc. Instrumental uncertainty of $Q_{z}$ is mainly owing to the divergence of the incident and exit Soller slits, estimated to be in the magnitude of $0.5^{\circ}$. The broadening due to the finite size of the crystal domain predicted from the Debye-Scherrer formula is assumed to be less than $1^{\circ}$ in view of layer thickness and the domain size determined with atomic force microscopy is greater than $10 \mathrm{~nm}$. The distribution of crystal orientation angles was $4^{\circ}-5^{\circ}$, which was estimated from the peak widths of $\left(\begin{array}{lll}1 & -1 & 1\end{array}\right)$ and $\left(\begin{array}{lll}0 & -2 & 1\end{array}\right)$ diffractions in the pole figure measurement. The spread of $Q_{z}$ is caused probably by the distribution of crystal orientations and was approximated as a Gaussian function with a full width at half maximum (FWHM) of $2 \sqrt{\ln 2} \Delta \alpha$; the intensity of GIXD is expressed as $I=I_{0} \exp \left(-(\alpha / \Delta \alpha)^{2}\right)$, where $\alpha$ is calculated from the relation of $\tan \alpha=Q_{z} / \sqrt{Q_{x}^{2}+Q_{y}^{2}}$.

The diffraction pattern simulated using the crystal structure data of Campbell et al. ${ }^{7}$ and Gaussian function with FWHM of $6.7^{\circ}$ is in good agreement with the experimental

patterns as shown in Fig. 1. 
TABLE I. Lattice parameters for the bulk phase pentacene thin film together with the literature data: single crystal x-ray diffraction (Ref. 7), and powder x-ray diffraction (Refs. 5 and 17).

\begin{tabular}{lllllllll}
\hline \hline & $a(\mathrm{~nm})$ & $b(\mathrm{~nm})$ & $c(\mathrm{~nm})$ & $\alpha(\mathrm{deg})$ & $\beta(\mathrm{deg})$ & $\gamma(\mathrm{deg})$ & $V\left(\mathrm{~nm}^{3}\right)$ & $d(001)(\mathrm{nm})$ \\
\hline This work & 0.608 & 0.790 & 1.584 & 112.7 & 101.3 & 85.7 & 0.688 & 1.437 \\
Ref. 7 & 0.606 & 0.790 & 1.601 & 112.6 & 101.9 & 85.8 & 0.692 & 1.45 \\
Ref. 17 & 0.6079 & 0.7893 & 1.591 & 112.71 & 101.58 & 85.60 & 0.6899 & 1.442 \\
Ref. 5 & 0.6485 & 0.7407 & 1.4745 & 77.25 & 85.72 & 80.92 & 0.6816 & 1.437 \\
\hline \hline
\end{tabular}

The diffraction pattern of the acetone-treated film is in particularly good agreement with the simulated one. In contrast, that of the thermally treated sample does not agree with it in intensity in high $Q$ region. We believe that the thermally treated film contains domains with their $c^{*}$ not normal to the substrate surface, because $(00 l)$ diffraction peaks are observed in this sample. The amount of the domains of these misorientations may be small judging from the results of the orientation measurements. Actually all the observed peaks could be indexed on the basis of the crystal structure reported by Campbell et al. ${ }^{7}$

In the same way we simulated a GIXD pattern using the cell parameters and structure model reported by Mattheus et $a l .{ }^{2,5}$ Even the peak positions that depend only on cell parameters were not reproduced. It is therefore clear that our bulk phase pentacene film has a completely different structure from that proposed by these authors. On the other hand, the cell parameters measured with $\mathrm{x}$-ray diffraction of a polycrystalline sample from vapor growth ${ }^{17}$ are close to ours.

As a result, we calculated cell parameters of the bulk phase from our data using $\gamma^{*}=90^{\circ}$, the angle between the reciprocal lattice vectors $\mathbf{a}^{*}$ and $\mathbf{b}^{*}$. The value of $\gamma^{*}$ can be estimated as $90^{\circ} \pm 0.5^{\circ}$ based on the absence of either $(h 00)$ or $(0 k 0)$ peaks with odd $h, k$ values or any splitting of the ( $h k 0)$ peaks within our experimental resolution. ${ }^{18}$ The cell parameters obtained are listed in Table I together with the reported results.

In summary, we examined bulk phase pentacene films on $\mathrm{SiO}_{2}$ using GIXD. The sample films were obtained using two reported methods. The obtained diffraction patterns of the two samples are mostly similar with each other, confirming that both films are the same in structure. The $\omega$ and $\phi$ scans and the pole figure measurements show that the $a b$ plane of the lattice is parallel to the substrate surface and its orientation around the $c^{*}$ axis is random. GIXD patterns were simulated for comparison of our results with the crystal structure data available in literature, assuming that the crystal is in a particular orientation and only the reciprocal lattice points of $Q_{z} \approx 0$ are detected. We conclude that the structure of the bulk phase pentacene thin film grown on a $\mathrm{SiO}_{2}$ substrate is the same as that of the single crystal reported by Campbell et al. $^{7}$

The authors thank T. Nemoto of Kyoto University for stimulating discussion on the pentacene film structures.
Thanks are also due to K. Inaba of Rigaku Corp. for helpful instructions in thin film $\mathrm{x}$-ray measurements. The authors would like to acknowledge T. Terasima and M. Takano of Kyoto University for kindly allowing us to use the $\mathrm{x}$-ray diffractometer. This work was supported by Grant-in-Aid for Young Scientists (B), No. 16750011, and Grant-in-Aid for Scientific Research on Priority Areas of Molecular Conductors, No. 15073214, from the Ministry of Education, Culture, Sports, Science and Technology of Japan.

${ }^{1}$ M. Pope and C. E. Swenberg, Electronic Processes in Organic Crystals and Polymers, 2nd ed. (Oxford University Press, New York, 1999).

${ }^{2}$ C. C. Mattheus, A. B. Dros, J. Baas, G. T. Oostergetel, A. Meetsma, J. L. de Boer, and T. T. M. Palstra, Synth. Met. 138, 475 (2003).

${ }^{3}$ I. P. M. Bouchoms, W. A. Schoonveld, J. Vrijmoeth, and T. M. Klapwijk, Synth. Met. 104, 175 (1999).

${ }^{4}$ D. Holmes, S. Kumaraswamy, A. J. Matzger, and K. P. C. Vollhardt, Chem.-Eur. J. 5, 3399 (1999).

${ }^{5}$ C. C. Mattheus, A. B. Dros, J. Baas, A. Meetsma, J. L. de Boer, and T. T. M. Palstra, Acta Crystallogr., Sect. C: Cryst. Struct. Commun. 57, 939 (2001).

${ }^{6}$ D. J. Gundlach, T. N. Jackson, D. G. Schlom, and S. F. Nelson, Appl. Phys. Lett. 74, 3302 (1999).

${ }^{7}$ R. B. Campbell, J. Monteath Robertson, and J. Trotter, Acta Crystallogr. 15, 289 (1962).

${ }^{8}$ T. Minakata, H. Imai, M. Ozaki, and K. Saco, J. Appl. Phys. 72, 5220 (1992).

${ }^{9}$ J. S. Wu and J. C. H. Spence, J. Appl. Crystallogr. 37, 78 (2004).

${ }^{10}$ L. F. Drummy and D. C. Martin, Adv. Mater. (Weinheim, Ger.) 17, 903 (2005).

${ }^{11}$ S. E. Fritz, S. M. Martin, C. D. Frisbie, M. D. Ward, and M. F. Toney, J. Am. Chem. Soc. 126, 4084 (2004).

${ }^{12}$ R. Ruiz, A. C. Mayer, G. G. Malliaras, B. Nickel, G. Scoles, A. Kazimirov, H. Kim, R. L. Headrick, and Z. Islam, Appl. Phys. Lett. 85, 4926 (2004).

${ }^{13}$ H. C. Yang, T. J. Shin, M. M. Ling, K. Cho, C. Y. Ryu, and Z. N. Bao, J. Am. Chem. Soc. 127, 11542 (2005).

${ }^{14}$ International Tables for Crystallography C, edited by A. J. C. Willson (Kluwer, Dordrecht, 1992), Sec. 2.3, p. 58.

${ }^{15}$ G. H. Vineyard, Phys. Rev. B 26, 4146 (1982).

${ }^{16}$ F. Izumi and T. Ikeda, Mater. Sci. Forum 321-324, 198 (2000).

${ }^{17}$ L. Farina, A. Brillante, R. G. Della Valle, E. Venuti, M. Amboage, and K. Syassen, Chem. Phys. Lett. 375, 490 (2003).

${ }^{18}$ The extinction rule for $(h 00)$ or $(0 k 0)$ peaks with odd $h, k$ values arises because the projection on the $a^{*} b^{*}$ plane has $p 2 g g$ symmetry where $\gamma^{*}$ is the right angle. Splitting of the $(h k 0)$ and $(h \bar{k} 0)$ peaks is imposed by the difference in the magnitude of the two vectors $\left|h \mathbf{a}^{*}+k \mathbf{b}^{*}\right|$ and $\left|h \mathbf{a}^{*}-k \mathbf{b}^{*}\right|$, which are equal when $\gamma^{*}=90^{\circ}$. This is the three-dimensional analog of the two-dimension case described in Ref. 12. 